\title{
CAPSULE COMMENTARIES \\ Capsule Commentary on Hoffman et al., Self-Reported Health Status Predicts Other-Cause Mortality in Men with Localized Prostate Cancer: Results from the Prostate Cancer Outcomes Study
}

\author{
Eva H DuGoff, PhD MPP \\ Department of Population Health Sciences, School of Medicine and Public Health, University of Wisconsin-Madison, Madison, WI, USA.
}

J Gen Intern Med 30(7):1000

DOI: $10.1007 / \mathrm{s} 11606-015-3188-7$

(c) Society of General Internal Medicine 2015

$\mathrm{R}$ outine screenings for prostate, breast, and colorectal cancers are generally not recommended for individual with limited life expectancy. ${ }^{1}$ Despite the consensus from specialty societies and clinical practice guidelines, these tests are commonly performed in individuals with high risk of mortality within 5 years. $^{2}$ The frequency with which these tests are overused speaks to the challenge of estimating life expectancy in clinical practice. To address this challenge, Hoffman and colleagues $^{3}$ offer an important contribution to the growing literature on how to best target clinical services based on life expectancy estimates. ${ }^{4,5}$

This study offers clinicians a practical aid - a nomogram - a tool that can be readily used in clinical practice to estimate the probability of other-cause mortality at 10 and 15 years in men. The nomogram's estimates are based on the 10 and 15-year other-cause mortality estimates from the population-based Prostate Cancer Outcomes Study (PCOS) cohort. The study estimates other-cause mortality as a function of the study participant's self-reported health status 6 months following prostate cancer diagnosis, race, and age. While the study aims to create a tool to facilitate screening decisions, it is important to note that the underlying study population of men diagnosed with prostate cancer may not be representative of all men eligible for screening.
This study provides a potentially new and practical tool to facilitate the appropriate targeting of prostate cancer screening to men most likely to benefit. Additional research is needed to examine the feasibility of the nomogram in practice, and its applicability to other types of cancer. If this type of tool can be reasonable integrated into practice, quality measures and Medicare payment policies can promote its use to promote appropriate cancer screenings and patient-shared decisionmaking.

Conflicts of Interest: The author has no conflicts of interest with the material in the article.

Corresponding Author: Eva H DuGoff, PhD MPP; Department of Population Health Sciences, School of Medicine and Public Health University of Wisconsin-Madison, 750 Highland Avenue, Madison, WI 53726-2336, USA (e-mail: dugoff@wisc.edu).

\section{REFERENCES}

1. Boodman SG. Concern is growing that the elderly get too many medical tests, in Kaiser Health News. Washington, DC; 2011.

2. Royce TJ, et al. Cancer screening rates in individuals with different life expectancies. JAMA Intern Med. 2014;174(10):1558-1565.

3. Hoffman RM, et al. Self-reported health status predicts other-cause mortality in men with localized prostate cancer: results from the prostate cancer outcomes study. J Gen Intern Med. 2015; doi:10. 1007/s11606-014-3171-8.

4. Cho H, et al. Comorbidity-adjusted life expectancy: a new tool to inform recommendations for optimal screening strategies. Ann Intern Med. 2013;159(10):667-676.

5. Lee SJ, et al. Individualizing life expectancy estimates for older adults using the Gompertz Law of Human Mortality. PLoS One. 2014;9(9):e108540. 